\title{
Free Excitonic Emission in Homoepitaxial Layers Grown on Bulk GaN Substrates
}

\author{
P. TAtarczaK ${ }^{a, *}$, H. Turski ${ }^{b}$ And A. WysmoŁeK ${ }^{a}$ \\ ${ }^{a}$ Faculty of Physics, University of Warsaw, L. Pasteura 5, 02-093 Warsaw, Poland \\ ${ }^{b}$ Institute of High Pressure Physics, Polish Academy of Sciences, \\ Sokołowska 29/37, 01-142 Warsaw, Poland \\ Doi: 10.12693/APhysPolA.139.300 \\ *e-mail: p.tatarczak@student.uw.edu.pl
}

\begin{abstract}
Variable temperature studies of free excitonic and phonon-assisted excitonic transitions in homoepitaxial gallium nitride $(\mathrm{GaN})$ layers grown on bulk substrates are presented. Photoluminescence measurements were performed in the temperature range between $4.2 \mathrm{~K}$ and $100 \mathrm{~K}$ in order to compare epilayers grown on substrates with different polarities. Surprisingly, one of the investigated samples exhibits unique properties which have not been reported for homoepitaxial GaN before - the low temperature free excitonic emission is more intense than neutral donor bound excitonic transitions. As expected for high quality homoepitaxial GaN layers, the excitonic spectrum of this sample consists of narrow spectral lines. Moreover, the shape of a longitudinal optical phonon replica of the free excitonic emission suggests a very low concentration of point defects involved in exciton scattering. This hypothesis concerning a high purity of the sample, is also corroborated by a very low signal of donor bound excitons. However, an observed overall optical inferiority in terms of photoluminescence intensity also suggests the appearance of non-radiative recombination channels which selectively affect radiative exciton recombination.
\end{abstract}

topics: GaN, homoepitaxy, photoluminescence, excitons

\section{Introduction}

GaN as a wide, direct band gap semiconductor has shown great potential in diverse optoelectronic devices [1, 2]. Although homoepitaxial GaN is, amongst others, widely used in light-emitting diodes (LEDs) and laser diodes (LDs), new methods for improving the quality of this material are still required. Especially promising is the growth along the [000-1] nitrogen polar (N-polar) direction, which is particularly important for applications in the novel type of lasers and high power LEDs with favorable built-in field alignment $[3,4]$. In these structures, the direction of built-in electric fields contributes to a better charge confinement in the active region of LEDs and LDs, which is crucial for the improvement of the optical efficiency of these devices. On the other hand, the growth along the N-polar direction is known to suffer from severe technological challenges, e.g., high oxygen and other defects incorporation as well as chemical instability of the surface which affect the efficiency of optoelectronic devices [5]. However, despite these difficulties, the successful growth of high quality homoepitaxial layers on N-polar substrates has recently been demonstrated [6].

In this report, we present a study of free excitonic emission in smooth homoepitaxial GaN layers grown on N-polar and Ga-polar substrates. It was found that the relative intensity of the free excitonic emission and the radiative recombination due to bound excitons depends on growth parameters. The results are discussed in terms of point defects and/or impurity scattering of free excitons. A comparison of photoluminescence (PL) signals of epilayers grown on substrates of different polarities suggests the appearance of two types of defects in homoepitaxial GaN. One of them is the well-known radiative recombination centers which results in, e.g., donor bound excitonic emission. Defects of the second type could be associated with non-radiative recombination centers which capture free excitons making radiative recombination channels ineffective. This, in turn, results in a drastic reduction of total excitonic emission. So far, a low intensity of bound excitons emission has been recognized as an indicator of the high purity of GaN structures but the presence of non-radiative centers has not been discussed in the literature in the context of excitonic emission in homoepitaxial GaN.

\section{Experimental details}

The investigated homoepitaxial GaN layers are grown by plasma-assisted molecular beam epitaxy (PAMBE) on bulk substrates with different polarities with low $\left(0.8^{\circ}\right)$ and high $\left(4.0^{\circ}\right)$ miscut angles under standard gallium-rich conditions [2] in the 
same epitaxial process. In this paper, we focus on the layer grown on a N-polar substrate with a low miscut $\left(0.8^{\circ}\right)$ angle which exhibits unique properties. This sample is compared with a standard high quality GaN layer grown on a Ga-polar substrate with a low miscut $\left(0.8^{\circ}\right)$ angle. The thickness of both layers is about $1 \mu \mathrm{m}$.

$\mathrm{PL}$ was obtained using a $\mathrm{He}-\mathrm{Cd}$ laser operating at $325 \mathrm{~nm}$. Spectra were acquired using a $0.5 \mathrm{~m}$ T64000 Horiba-Jobin-Yvon spectrometer, working in a single grating mode, using a nitrogencooled Symphony CCD camera. The spectral resolution provided by the experimental setup was about $0.5 \mathrm{meV}$. The PL measurements were performed in the range between $4.2 \mathrm{~K}$ and $100 \mathrm{~K}$, however, in this communication, we focus on the results obtained at $4.2 \mathrm{~K}$ and $40 \mathrm{~K}$.

\section{Results and discussion}

The PL spectrum of the investigated homoepitaxial GaN layer measured at $4.2 \mathrm{~K}$ is shown in Fig. 1. It consists of three free excitonic transitions $\left(\mathrm{X}_{A}\right.$, $\mathrm{X}_{B}, \mathrm{X}_{C}$ ) originating from the splitting of the valence band into 3 subbands, as expected in wurtzite type semiconductors, neutral donor bound exciton transitions $\left(\mathrm{D}^{0} \mathrm{X}_{A}\right)$ which are typically assigned to oxygen and silicon donors [7-10], LO phonon replica of free exciton transitions (1-LO) [11, 12] and 2 peaks signed as $R_{4}$ and $R_{5}$. These later transitions are in a perfect agreement with values of the 4th and 5th LO phonon replica of the $\mathrm{He}-\mathrm{Cd}$ laser line (3.448 eV and $3.356 \mathrm{eV}$, respectively). The observation of the Raman signal on top of PL suggests a very low total intensity of PL, which usually hinders the Raman scattering measurements. The low temperature PL spectra in the excitonic region are typically dominated by neutral donor bound exciton transitions due to the conservation of momentum and thermalization process of free excitons. In the light of the above mentioned, an intensity ratio $\mathrm{X}_{A} / \mathrm{D}^{0} \mathrm{X}_{A}$ larger than 1 suggests an extremely low point defect concentration. Very narrow $\mathrm{D}^{0} \mathrm{X}_{A}$, $\mathrm{X}_{A}$, and $\mathrm{X}_{B}$ lines (full widths at half maximum are about $0.6,3,4 \mathrm{meV}$, respectively) also indicate a high quality crystal structure and low dislocation density.

Complementary information about the purity of the sample can be obtained from an analysis of the shape of the 1-LO replica of free exciton transitions. It has been already shown that at higher temperatures a phonon replica of the excitonic region is mostly related to free excitons [13]. According to Permogorov's theory for exciton-phonon coupling [14], the 1-LO line shape is given by

$$
\begin{aligned}
I & \sim J \sqrt{\varepsilon_{A}} \exp \left(-\frac{\varepsilon_{A}}{k_{\mathrm{B}} T}\right) W\left(\varepsilon_{A}\right) \\
& +\sqrt{\varepsilon_{B}} \exp \left(-\frac{\varepsilon_{B}}{k_{\mathrm{B}} T}\right) W\left(\varepsilon_{B}\right),
\end{aligned}
$$

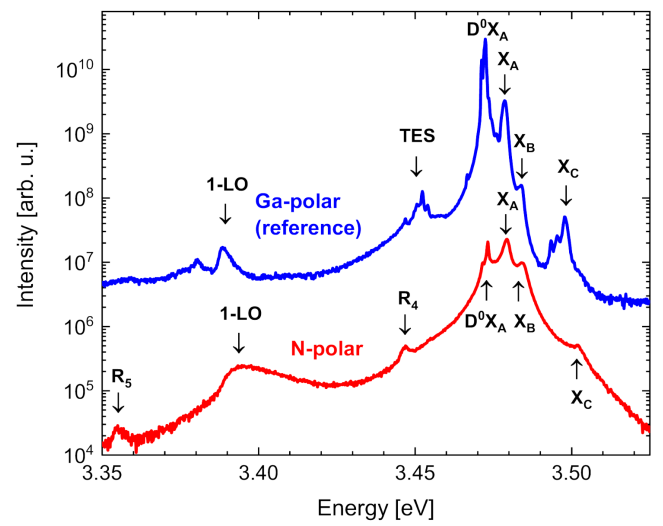

Fig. 1. Measured PL spectrum of the investigated homoepitaxial GaN layer (red) at $4.2 \mathrm{~K}$. Luminescence spectrum of an analogous layer grown on a Ga-polar substrate is shown as a reference (blue). $\mathrm{X}_{A}, \mathrm{X}_{B}, \mathrm{X}_{C}-$ free excitons, $\mathrm{D}^{0} \mathrm{X}_{A}-\mathrm{X}_{A}$ excitons bound to neutral donors, TES - two electron satellites $[9,10], 1-\mathrm{LO}-1$ st LO phonon replica of an excitonic region, $\mathrm{R}_{4}, \mathrm{R}_{5}$ - Raman LO bands excited by the incident laser.

where $I$ is the phonon replica intensity, $k_{\mathrm{B}}$ is the Boltzmann constant, $T$ is the temperature, $\varepsilon_{A}$ and $\varepsilon_{B}$ are kinetic energies of free excitons $\mathrm{X}_{A}$ and $\mathrm{X}_{B}\left(\mathrm{X}_{C}\right.$ is not included due to the low intensity), $J$ is related to the ratio of $\mathrm{X}_{A}$ and $\mathrm{X}_{B}$ intensities and differences in the coupling strength of LO phonons to excitons. The probability of the phonon-assisted free exciton recombination is denoted as $W(\varepsilon)$ and is usually proposed to be described by a simple power law $W(\varepsilon) \sim \varepsilon^{P}$. The kinetic energy $\varepsilon_{A}$ of the free exciton $\mathrm{X}_{A}$ can be expressed by the free exciton energy $E_{A}\left(\mathrm{X}_{A}\right.$ position in the measured spectrum), LO phonon energy $E_{\mathrm{LO}}$ and observed photon energy $E$ as follows:

$$
\varepsilon_{A}=E+E_{\mathrm{LO}}-E_{A} \text {. }
$$

A similar analysis can be performed for free excitons $\mathrm{X}_{B}$ as the formula for $\varepsilon_{B}$ is analogous. Previous results indicate a good agreement between the experimental data and the proposed theory with $P=1$ for heteroepitaxial GaN of high purity grown on $6 \mathrm{H}-\mathrm{SiC}$ [11] and with $P=0$ for homoepitaxial GaN layers [12]. In the case of heteroepitaxial layers, one can expect a lattice mismatch at the interface of a GaN epilayer and a substrate. This results in a higher dislocation density and fluctuation of compressive strain which leads to a broadening of the observed spectral lines (the full width at half maximum of the bound exciton line in structures measured in [11] is about $5 \mathrm{meV}$ ). However, the linear dependence of $W(\varepsilon)$ of free excitons kinetic energies indicates that such defects do not have a considerable influence on exciton scattering. Homoepitaxial GaN layers are known to result in quite a different behavior - they exhibit very narrow spectral lines which can be observed thanks to the absence of strain and dislocations [15]. 


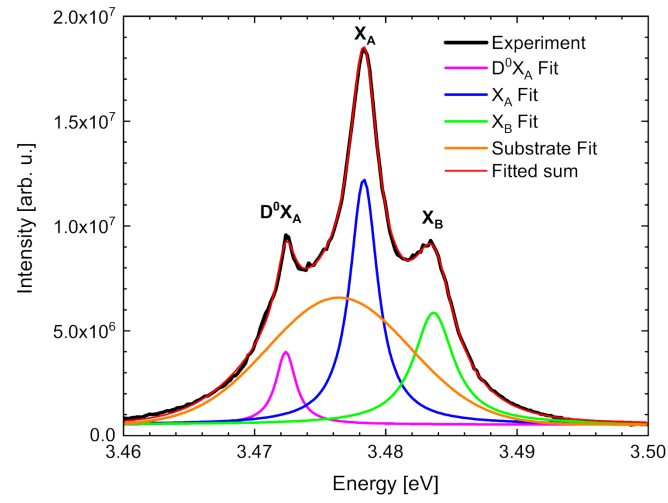

Fig. 2. Measured PL spectrum for the investigated homoepitaxial GaN layer at $40 \mathrm{~K}$ in the excitonic region.

However, the experimentally observed independence of $W(\varepsilon)$ on the free exciton kinetic energy in homoepitaxial GaN layers can be explained only by assuming a high concentration of point defects and impurities which have been shown to introduce new states into the free exciton scattering process $[12,14]$.

Figure 2 and Fig. 3a show the PL spectra of the excitonic and first phonon replica regions for investigated homoepitaxial GaN layer at $40 \mathrm{~K}$. Due to the low PL signal of the epilayer, the impact of the substrate on the observed spectra is significant. To account for PL emission associated with the recombination inside the substrate, the epilayer was removed on a part of the wafer. Reactive ion etching (1.5 $\mu \mathrm{m}$ deep) was used with a part of the sample protected from etching by a photoresist. This way the PL signal from both parts: the epilayer and substrate could be easily collected and compared quantitatively. Approximating $\mathrm{X}_{A}$ and $\mathrm{X}_{B}$ exciton lines with Lorentzian curves, the obtained peak positions energies are $E_{A}=3.478 \mathrm{eV}$ and $E_{B}=3.483 \mathrm{eV}$, respectively. Assuming that the coupling strength of the LO phonon to the free exciton is the same for $\mathrm{X}_{A}$ and $\mathrm{X}_{B}, J$ is the intensity ratio $\mathrm{X}_{A} / \mathrm{X}_{B}$ equal to 2.19. Figure 3a shows results of the fitting of (1) to experimental data with obtained values of $E_{A}, E_{B}, J$, and by putting $E_{\mathrm{LO}}=91.8 \mathrm{meV}$ for $P=0$ and $P=1$. The only fitting parameter is the amplitude since $\mathrm{X}_{A}$ and $\mathrm{X}_{B}$ peaks widths are considered. The fitted curve for $P=1$ follows experimental data almost perfectly. This suggests an extremely low point defects concentration - significantly lower than in homoepitaxial GaN layers investigated in [12] and in the reference sample, for which the first phonon replica line can be approximately reproduced using $P=0.5$ (see Fig. $3 \mathrm{~b}$ ). The value of $P=0.5$ indicates that the quality of the standard, reference GaN epilayer is better than in previous results [12] in terms of point defects causing scattering of free excitons but it is still much lower than in the investigated GaN layer grown on N-polar substrate.

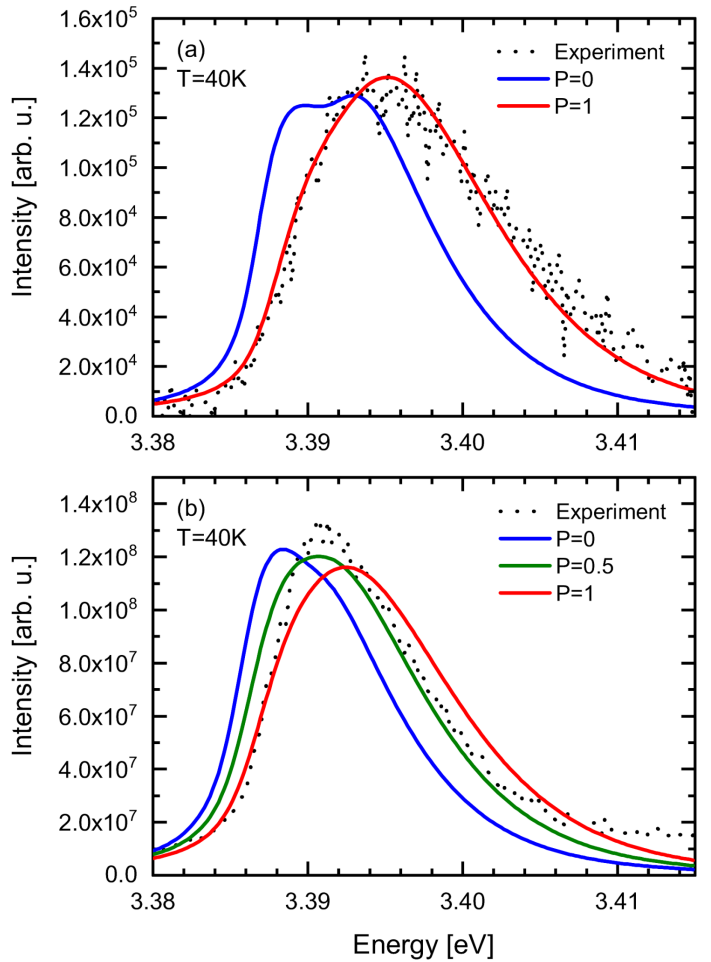

Fig. 3. Luminescence spectrum of the first phonon replica measured at $40 \mathrm{~K}$ for (a) the investigated, (b) the reference homoepitaxial GaN layer.

The obtained results strongly suggest that we deal with the extraordinarily pure GaN layer. However, focusing only on the investigated sample can lead to a misinterpretation of the measured spectra. The PL intensity of the studied GaN layer at $4.2 \mathrm{~K}$ is about 100-1000 times lower than the PL intensity of the reference sample which contains many donors indicated by a high $\mathrm{DX}_{A}$ peak (see Fig. 1). The relatively low PL intensity suggests a dominant role of non-radiative processes in the investigated homoepitaxial GaN layer which remains to be verified in the future by time resolved PL measurements. In the case of the excitonic emission in high purity GaN layers, long decay times are expected which otherwise should be shortened for samples with higher defect concentration.

\section{Conclusions}

Photoluminescence emission in a state-of-the-art homoepitaxial GaN layer was studied at $4.2 \mathrm{~K}$ and $40 \mathrm{~K}$. As expected for homoepitaxial growth, we observe narrow spectral lines which suggest a low dislocation density and a lack of strain. Furthermore, at first glance, the investigated GaN epilayer exhibits some properties typical for heteroepitaxial growth. The high intensity ratio $\mathrm{X}_{A} / \mathrm{D}^{0} \mathrm{X}_{A}$ indicates a low concentration of point defects in the layer grown on a N-polar GaN substrate. This hypothesis is further supported by the shape of the phonon-assisted free exciton transition line. 
An observed linear dependence of $W(\varepsilon)$ on the kinetic energies of free excitons in homoepitaxial GaN layers has not been reported up to now. In the literature, $P=1$ is regarded as an indicator of excellent optical properties and the absence of point defects. However, the overall low intensity of the measured PL signal seems to contradict this thesis. Such a low emission can originate from the presence of defects that do not change the line shape of 1-LO peak but are responsible for non-radiative recombination processes. The hypothesis concerning a new class of defects in GaN grown on N-polar substrates gives us a new research perspective that needs to be further verified in future studies including time resolved photoluminescence.

\section{References}

[1] S. Nakamura, Rev. Mod. Phys. 87, 1139 (2015).

[2] C. Skierbiszewski, H. Turski, G. Muziol, M. Siekacz, M. Sawicka, G. Cywiński, Z.R. Wasilewski, S. Porowski, J. Phys. D Appl. Phys. 47, 073001 (2014).

[3] J. Verma, J. Simon, V. Protasenko, T. Kosel, H.G. Xing, D. Jena, Appl. Phys. Lett. 99, 171104 (2011).

[4] H. Turski, S. Bharadwaj, H.G. Xing, D. Jena, J. Appl. Phys. 125, 203104 (2019).

[5] C. Lund, S. Nakamura, S.P. DenBaars, U.K. Mishra, S. Keller, Semicond. Sci. Technol. 34, 075017 (2019).
[6] Y. Cho, Z. Hu, K. Nomoto, H.G. Xing, D. Jena, Appl. Phys. Lett. 110, 253506 (2017).

[7] K.P. Korona, A. Wysmołek, K. Pakuła, R. Stępniewski, J.M. Baranowski, Appl. Phys. Lett. 69, 788 (1996).

[8] K. Pakuła, A. Wysmołek, K.P. Korona et al., Solid State Commun. 97, 919 (1996).

[9] A. Wysmolek, K.P. Korona, R. Stępniewski et al., Phys. Rev. B 66, 245317 (2002).

[10] A. Wysmołek, R. Stępniewski, M. Potemski, B. Chwalisz-Piętka, K. Pakuła, J.M. Baranowski, D.C. Look, S.S. Park, K.Y. Lee, Phys. Rev. B 74, 195205 (2006).

[11] D. Kovalev, B. Averboukh, D. Volm, B.K. Meyer, Phys. Rev. B 54, 2518 (1996).

[12] I.A. Buyanova, J.P. Bergman, B. Monemar et al., Solid State Commun. 105, 497 (1998).

[13] A. Wysmołek, P. Łomiak, J.M. Baranowski, K. Pakuła, R. Stępniewski, K.P. Korona, I. Grzegory, M. Boćkowski, S. Porowski, Acta Phys. Pol. A 90, 981 (1996).

[14] S. Permogorov, Excitons, Eds. E.I. Rashba, M.D. Sturge, North-Holland, Amsterdam 1982, Ch. 5.

[15] K. Kornitzer, T. Ebner, K. Thonke, R. Sauer, C. Kirchner, V. Schwegler, M. Kamp, M. Leszczynski, I. Grzegory, S. Porowski, Phys. Rev. B 60, 1471 (1999). 